\title{
The 1997 event in the Crab Pulsar in X-rays (Research Note)
}

\author{
M. Vivekanand
}

\begin{abstract}
No. 24, NTI Layout 1st Stage, 3rd Main, 1st Cross, Nagasettyhalli, 560094 Bangalore, India e-mail: viv.maddali@gmail.com
\end{abstract}

Received 10 July 2015 / Accepted 29 December 2015

\begin{abstract}
Context. In October 1997, radio pulses from the Crab Pulsar underwent abnormal delay. This was reported by two radio observatories, both of which explained this frequency dependent and time varying delay as being due to refractive effects of ionized shells in the Crab Nebula. Both groups also noted that, curiously and confusingly coincident with the frequency dependent delay, the Crab Pulsar also underwent an unusual slowing down, which they believed to be unrelated to the Crab Nebula and instead intrinsic to the Crab Pulsar, resulting in an additional delay that was frequency independent. However, it now appears that one of the groups attributes the frequency independent delay also to refractive effects.

Aims. This work aims to verify whether at least a part of the frequency independent delay is indeed due to intrinsic slowing down of the Crab Pulsar.

Methods. Timing analysis of the Crab Pulsar's October 1997 event has been done in X-rays, which are not delayed by the refractive and diffractive effects that affect radio waves; at X-rays only the intrinsic slowing down should contribute to any observed delay. Data mainly from the PCA instrument aboard the RXTE satellite have been used, along with a small amount of data from the PDS instrument aboard the BeppoSAX satellite.

Results. Analysis of the X-ray data, using the very accurate reference timing model derived at radio frequencies, strongly supports the intrinsic slowing down hypothesis. Analysis using the reference timing model derived self-consistently from the limited X-ray data, which is less accurate, is not completely unambiguous regarding the above two hypotheses, but provides reasonable support for the intrinsic slowing down hypothesis.

Conclusions. A significant fraction of the frequency independent delay during the October 1997 event is indeed due to intrinsic slowing down of the Crab Pulsar.
\end{abstract}

Key words. pulsars: general - pulsars: individual: Crab Pulsar

\section{Introduction}

Radio pulses from pulsars undergo several kinds of transformation while traveling through the ionized interstellar medium, such as dispersion, pulse scatter broadening, scintillation, and delayed arrival of pulses; see Manchester \& Taylor (1977) and Lyne \& Smith (2006) for pedagogical reviews; see also Backer et al. (2000) and Lyne et al. (2001) and references therein for technical details. The Crab Pulsar in particular displays extreme forms of such activity from time to time (Smith \& Lyne 2000). However, the event of late October 1997 in the Crab Pulsar stands out in that it was simultaneously accompanied by a dramatic slowing down of the Crab Pulsar (Backer et al. 2000; Smith \& Lyne 2000; Lyne et al. 2001); it slowed down by $\approx 1.2 \mathrm{~ms}$ in $\approx 10$ to 20 days. In comparison, the Crab Pulsar wanders in delay by about $\approx 0.3 \mathrm{~ms}$ over a month owing to timing noise (Backer et al. 2000), and is delayed by about $\approx 0.0007 \mathrm{~ms}$ over 20 days owing to systematic loss of rotational energy. Backer et al. (2000) and Smith \& Lyne (2000) note the curious and confusing coincidence of the dramatic slowing down (which is presumed to be due to factors internal to the Crab Pulsar) with the dispersive, diffractive, and refractive phenomena (which are presumed to be due to factors related to the Crab Nebula). Both groups developed their respective models to explain the dispersive, diffractive, and refractive phenomena in terms of moving ionized screens within the Crab Nebula (Backer et al. 2000; Smith \& Lyne 2000; Lyne et al. 2001.

More recently, however, Smith et al. (2011) have suggested that there was no intrinsic slowing down of the Crab Pulsar during the October 1997 event; they attribute the entire nondispersive, frequency independent delay of $1.2 \mathrm{~ms}$ to ray paths in highly localized electron clouds in the Crab Nebula. A similar hint appears in the work of Wong et al. (2001), in the last sentence of their Sect. 3.4. A perspective of the problem is provided by Fig. 2a in Smith et al. (2011), which shows the variation of frequency independent timing residuals of the Crab Pulsar during the October 1997 event. The event being discussed here refers to the dramatic slowing down of the Crab Pulsar at around MJD 50750 (29 October 1997), which manifests as increasing timing residuals. A glitch occurred at MJD $50812.59 \pm 0.01$ (Espinoza et al. 2011), which is not believed to be connected to the October 1997 event (Backer et al. 2000; Smith et al. 2011).

This work attempts to verify, at least qualitatively, whether the Crab Pulsar intrinsically slowed down or not during late October 1997 by analyzing X-ray data for that epoch. X-ray propagation is not affected by passage through ionized media, unlike radio waves; so X-ray timing of the Crab Pulsar should show only the unusual intrinsic slowing down, if any, and not 
other delays. On the other hand, X-ray data is very sparse (i.e., poorly sampled in time) compared to radio data. Only four X-ray observatories capable of timing the Crab Pulsar observed it during that epoch. Out of the four, ROSAT and ASCA did not yield useful data. BeppoSAX provided useful data for only one epoch. All other data came from the RXTE observatory; however, these observations occurred on average once a week or once every two weeks, whereas the radio observations took place at least once every day. Therefore, quantitative comparison between the X-ray and radio results may not be constraining.

\section{Observations}

\subsection{RXTE PCA observations}

The RXTE data are obtained from the Proportional Counter Array (PCA; Jahoda et al. 1996) aboard the RXTE observatory. The PCA consists of five proportional counter units (PCUs) operating in the $2-60 \mathrm{keV}$ range, having a field of view of $1^{\circ}$ in the sky, and a time resolution of $1 \mu$ s (see "The ABC of XTE" guide on the RXTE website ${ }^{1}$ ). The first observation was on 20 July 1997 (MJD 50650, ObsID 10200-01-12-00) and the last observation was on 11 April 1998 (MJD 50914, ObsID 30133-01-08-00); there were a totoal of 28 ObsIDs. The closest observation preceding this duration was on 9 May 1997 (MJD 50 577, ObsID 10200-01-13-00), which was not included in the analysis owing to the $\approx 73$-day gap. The last observation for the analysis was chosen so as to sufficiently represent the glitch behavior. These data were obtained in the Generic Single Bit mode, having the configurations $S B \_250 u s \_0 \_13 \_2 s$ and $S B \_250 u s \_14 \_249 \_2 s$. Both configurations accumulated photon events into time bins of size $244.14 \mu \mathrm{s}$; the former mode combined the first 14 energy channels into a single channel, while the latter combined the next 236 energy channels. Of the 28 ObsIDs, 22 had all five PCUs switched on, while 3 had four PCUs switched on, and 3 had only three PCUs switched on.

\subsection{BeppoSAX PDS observation}

The BeppoSAX observatory observed the Crab Pulsar on 8 October 1997 (MJD 50 729, ObsID 20795004) using, among other narrow field instruments (NFIs), the Phoswich Detector System (PDS; Frontera et al. 1997). The PDS consists of four $\mathrm{NaI}(\mathrm{Tl}) / \mathrm{CsI}(\mathrm{Na})$ phoswich scintillation photon counters grouped into two pairs, which rock on and off the source in the sky, similar to the HEXTE instrument of RXTE. It covers the energy range $15-300 \mathrm{keV}$ and has a time resolution of $16 \mu$ s (see the "Cookbook for BeppoSAX NFI Spectral Analysis" guide on the BeppoSAX website ${ }^{2}$ ). This lone BeppoSAX observation was considered important since its epoch is close to the dramatic slowdown of the Crab Pulsar. BeppoSAX also observed the Crab Pulsar on 6 April 1998 (MJD 50 909), which falls in the glitch part of the data in Fig. 1; this data could not be analyzed owing to the lack of housekeeping files.

\section{Analysis}

Vivekanand (2015) has described in comprehensive detail the timing analysis for the Crab Pulsar using the HEXTE instrument. The method of analysis differs from instrument to instrument

\footnotetext{
1 http://heasarc.gsfc.nasa.gov/docs/xte/data_analysis. html

2 http://wWw.asdc.asi.it/bepposax/software/index.html
}

mainly up to the stage of referring the photon arrival times to the solar system barycenter.

When combining data from different instruments, the keyword TIMEPIXR is important. All data used in this work have TIMEPIXR $=0$. The pulse arrival epochs determined by the tool efold have been corrected by the values $+122 \mu$ s and $+8 \mu$ s for the instruments PCA/SB and PDS, respectively, as per the prescription in the document "RXTE Absolute Timing Accuracy" on the RXTE website ${ }^{3}$.

\subsection{RXTE PCA analysis details}

Data for each of the two configurations were analyzed separately until the very end. It was found that in 25 of the 28 ObsIDs, there was negligible difference in arrival times of the two configurations (less than a milliperiod), so their average was used for the final analysis. The same was done for two of the three remaining ObsIDs, in which the difference was -477.9994 periods, which is very close to an even number of periods. In the third ObsID, the difference was -89.0000 periods, which is an odd number of periods, so averaging the two arrival times will result in an error of half a period; so the arrival time of one of the configurations was used in this case. For more details of analysis for this instrument see Vivekanand (2015).

\subsection{BeppoSAX PDS analysis details}

BeppoSAX PDS data was analyzed using FTOOLS version 5.0 and SAXDAS version 2.3.1. The following data was downloaded for ObsID 20795004: the cleaned and linearized event file, the GTI file, and the housekeeping file. Using the housekeeping file, an additional system related GTI was created using the following selections: (a) Earth occultaion angle, EARTH_ANG $>14^{\circ}$; (b) time since the peak of the last South Atlantic Anomaly passage, TIME_SINCE_SAA > $30 \mathrm{~min}$ or TIME_SINCE_SAA $<0$ min; (c) star tracker configuration, ID STR_CONF $>=4$ and STR_CONF $<=6$; see the "Aspect Reconstruction" section of "BeppoSAX Cookbook" on the BeppoSAX website ${ }^{4}$. In summary, GTI selection criteria are as per standard procedures, with additional constraints on the three parameters mentioned above. After selecting photons based on this GTI, the light curve was obtained using the tool lcurve; based on this light curve, the data beyond $70 \mathrm{ks}$ from the start of the data file were excluded from further analysis. Then photons were selected in the energy range 15 to $220 \mathrm{keV}$. Finally the tool baryconv was used to refer photon arrival times to the solar system barycenter. Further analysis was similar to that of the PCA instrument of RXTE.

\section{Results for the October 1997 event using the radio reference timing model}

In this section the X-ray data are analyzed using the reference timing model of Backer et al. (2000), given in their Table 1; this is used to check that the same phenomenon is being observed that was observed by radio astronomers. The Jodrell group have not published their reference timing model. Figure 1 shows the result of using TEMPO2 along with the parameters in Table 1 of Backer et al. (2000) as constant input (i.e., without

\footnotetext{
3 http://heasarc.gsfc.nasa.gov/docs/xte/abc/time.html 4 http://www.asdc.asi.it/bepposax/software/cookbook/ attitude.html
} 


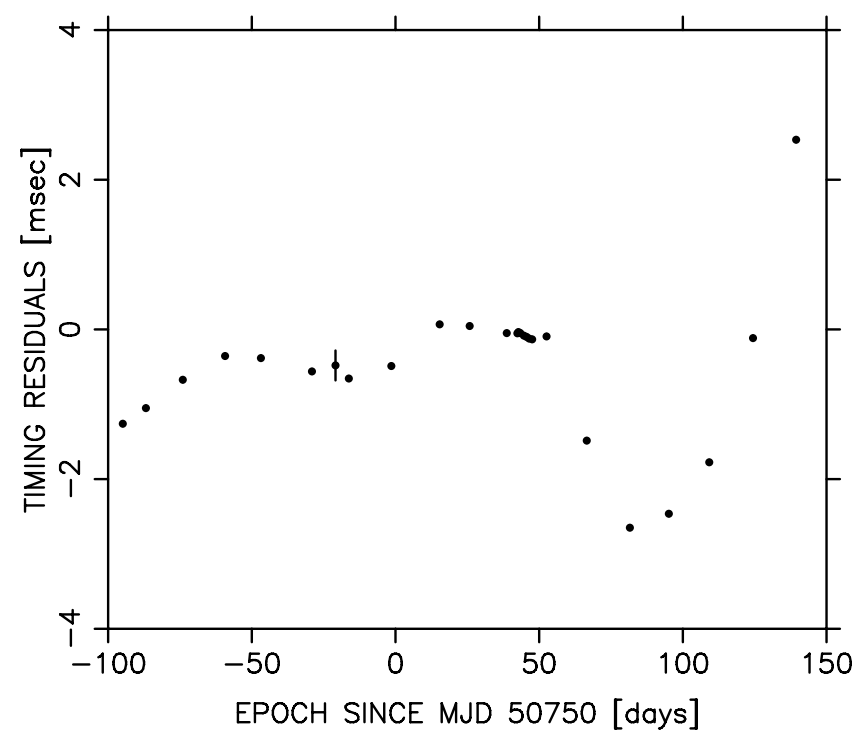

Fig. 1. Result of using TEMPO2 on all data with the parameters given in Table 1 of Backer et al. (2000) as constant input (i.e., without any fitting). The lone BeppoSAX data point is at epoch $\approx-21$ days with a larger error bar. We note that no artificial phase jumps (using PHASE \pm 1 in the input file to TEMPO2) are required, pointing to the robustness of the radio reference timing model used.

any fitting) for the X-ray data from the PCA/HEXTE and from PDS/BeppoSAX. This figure is remarkably similar to Fig. 2a in Smith et al. (2011). The slowing down of the 1997 event and the glitch behavior are clearly evident, including the negative slope in residuals between the two epochs. In Fig. 1, the Crab Pulsar slowed down by $\approx 0.7 \pm 0.1 \mathrm{~ms}$ over a duration of $\approx 30$ days around MJD 50750. This is smaller than, but consistent with, the findings of Backer et al. (2000), Smith \& Lyne (2000), and Lyne et al. (2001). Furthermore, the delay observed here is about half of that observed at radio frequencies. We therefore conclude that the Crab Pulsar indeed slowed down intrinsically during the 1997 event.

\section{Results for the October 1997 event using the reference timing model derived from $X$-rays}

In this section the X-ray data are analyzed using reference timing models that are derived self-consistently from the X-ray data itself. These data are not expected to be as accurate as the radio reference timing model owing to sparse X-ray timing data.

The first step is to identify precisely the "post-event and preglitch" duration in Fig. 1; this duration will be used to obtain the reference timing model for the entire data. Based on the independent identifications by Backer et al. (2000), Lyne et al. (2001), and Smith et al. (2011), which differ marginally from each other, for our purposes the post-event and pre-glitch duration can be safely identified as being from MJD 50758 to MJD 50812.

The second step is to fit the X-ray data in the above postevent and pre-glitch duration to a pulsar slowdown model (using TEMPO2 Hobbs et al. (2006)), which is conventionally taken as consisting of three parameters: rotation frequency $v$, its derivative $\dot{v}$, and its second derivative $\ddot{v}$; see Eq. (1) of Espinoza et al. (2011). Two such models are derived, which are shown as models 1 and 2 in Table 1 . Model 1 is obtained by a fit of the X-ray data in the post-event and pre-glitch duration using TEMPO2. Model 2 is an arbitrary but minor modification of model 1,
Table 1. TEMPO2 best fit parameters to the post-event and pre-glitch duration in Fig. 1, which is identified as being from MJD 50758 to MJD 50812

\begin{tabular}{lcc}
\hline \hline Parameter & Model 1 & Model 2 \\
\hline$v(\mathrm{~Hz})$ & $29.870335054(1)$ & 29.870335055 \\
$\dot{v}\left(10^{-10} \mathrm{~s}^{-2}\right)$ & $-3.75266(2)$ & -3.75265 \\
$\ddot{v}\left(10^{-20} \mathrm{~s}^{-3}\right)$ & $0.8(2)$ & 1.2 \\
\hline
\end{tabular}

Notes. $v$ is the rotation frequency of the Crab Pulsar at the reference epoch MJD 50797.5233442733588; $\dot{v}$ and $\ddot{v}$ are the first and second time derivatives of $v$, respectively, at the same epoch. The errors in brackets are in the last digit of each result. Both models use PCA data only, since the BeppoSAX data lies outside this range. While model 1 is obtained using TEMPO2, model 2 is an arbitrary modification of model 1 , for reasons explained in the text, which is why no errors are quoted for these parameters.

for reasons explained later. The rms residuals of the fit in the two models is $21 \mu \mathrm{s}$ and $68 \mu \mathrm{s}$, respectively. Within the errors both models are similar. The corresponding parameters from the Jodrell Bank monthly timing ephemeris of the Crab Pulsar ${ }^{5}$ (Lyne et al. 1993), referring to the reference epoch in Table 1, are $29.870335055 \mathrm{~Hz}$ and $-3.75274 \times 10^{-10} \mathrm{~s}^{-2}$, which compare very well with the estimates in Table 1.

The final step is to use TEMPO2 along with the parameters in Table 1 as constant input (i.e., without any fitting) for the entire data; the results are presented in the two panels of Fig. 2. The glitch behavior is clearly seen using both models, although it is not shown in the figure; qualitatively they are similar to the glitch behavior seen in Fig. 2a in Smith et al. (2011), while quantitative comparison is done in the following section. The occurrence of a glitch soon after the event of October 1997 in the Crab Pulsar is fortuitous, since its analysis in the current X-ray data validates the method of analysis for the event as well.

Unfortunately, the X-ray data analysis of this section is not unequivocal regarding the October 1997 event. Using Model 1, the pre-event X-ray timing residuals can be interpreted as a speeding up of the Crab Pulsar (top panel of Fig. 2), while using Model 2 they can be interpreted as a slowing down (bottom panel of Fig. 2). The most probable reason for this is the sparse (i.e., poorly sampled in time) X-ray data. Model 2 was obtained by trial and error, in the effort to ascertain whether the X-ray data could show an event behavior contrary to that obtained using Model 1. We therefore conclude from the above analysis that the X-ray data by itself cannot categorically determine whether the Crab Pulsar intrinsically slowed down during the event of October 1997.

However, a closer look at Fig. 2 reveals a detail. In both panels of Fig. 2, the first data point before the post-event and pre-glitch duration at epoch $\approx-1.43$ days is lower than the reference data (i.e., data later than this epoch in Fig. 2). In the bottom panel of Fig. 2 it is expected to be so anyway, but in the top panel of Fig. 2 it is lower by $0.30 \pm 0.04 \mathrm{~ms}$. Therefore, it appears that even Model 1 indicates a slowing down of the Crab Pulsar around the epoch -1.43 days, or equivalently at round MJD 50 750-1.43 = MJD 50748.57. It can therefore be concluded from the above analysis that although the X-ray data analysis of this section cannot categorically determine whether the Crab Pulsar slowed down during the event of October 1997, it offers reasonable support for that hypothesis.

http://www.jb.man.ac.uk/pulsar/crab.html 
Table 2. TEMPO2 best fit parameters to the post-glitch X-ray data.

\begin{tabular}{lcccc}
\hline \hline Parameter & Wong et al. (2001) & Backer et al. (2000) & Model 1 & Model 2 \\
\hline$t_{\mathrm{g}}$ (MJD) & $50812.9_{-1.5}^{+0.3}$ & & & \\
$\tau_{n}$ (days) & $2.9 \pm 1.8$ & & & \\
$\Delta v_{n}\left(10^{-7} \mathrm{~Hz}\right)$ & $2.4 \pm 0.6$ & $2.1 \pm 0.9$ & $2.1 \pm 0.9$ & $2.1 \pm 0.9$ \\
$\Delta v_{\mathrm{p}}\left(10^{-7} \mathrm{~Hz}\right)$ & $0.17 \pm 0.05$ & $0.28 \pm 0.06$ & $0.32 \pm 0.06$ & $0.26 \pm 0.06$ \\
$\Delta \dot{v}_{\mathrm{p}}\left(10^{-15} \mathrm{~s}^{-2}\right)$ & $-14.2 \pm 0.6$ & $-13 \pm 2$ & $-8 \pm 3$ & $-14 \pm 2$ \\
\hline
\end{tabular}

Notes. The second column lists the glitch parameters derived by Wong et al. (2001). Their values of glitch epoch $t_{\mathrm{g}}$ and decay time scale $\tau_{n}$ have been used as given constants. The derived glitch parameters are (a) jump in frequency that is exponentially recovered $\Delta v_{n}$; (b) permanent jump in frequency $\Delta v_{\mathrm{p}}$, and (c) permanent jump in frequency derivative $\Delta \dot{v}_{\mathrm{p}}$. The third to fifth columns list the values derived using the model of Backer et al. (2000), and models 1 and 2 in Table 1.

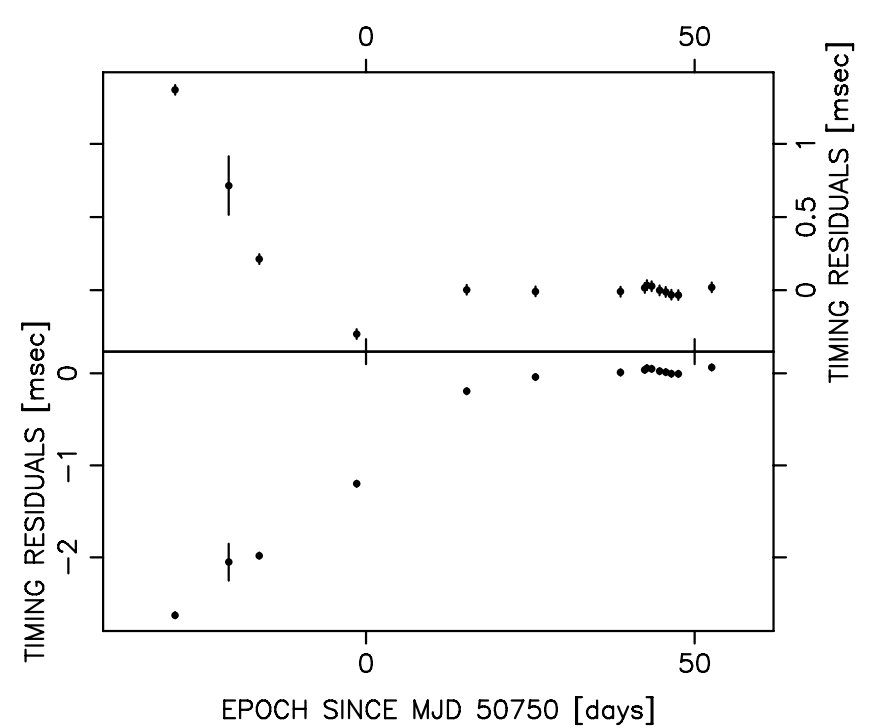

Fig. 2. Top panel: result of using TEMPO2 on the X-ray data with the parameters of model 1 in Table 1 as constant input (i.e., without any fitting). The plot is restricted to the post-event and pre-glitch duration, along with four data points before this duration, to better view the event itself. Bottom panel: same as top panel, but using the parameters of model 2 in 1.

In Figs. 1 and 2, the lone BeppoSAX timing residual differs by about $\approx 0.17 \pm 0.20 \mathrm{~ms}$ with respect to RXTE/PCA data, which is consistent with the formal error of $\approx 0.2 \mathrm{~ms}$ on this timing residual (Mineo et al. 2000). See also Cusumano (2003) and Nicastro et al. (2004) for details of the accuracy of the onboard clock of BeppoSAX. The lone BeppoSAX data point used in this work follows the RXTE timing residual variation in both Figs. 1 and 2, without having to artificially insert phase cycle corrections in TEMPO2. We believe that the lone BeppoSAX data point significantly enhances the confidence of the fits to the RXTE data since it is obtained from an entirely independent observatory.

\section{Results for the December 1997 glitch}

The results of the previous section depend critically upon the post-event and pre-glitch solutions of TEMPO2, which are listed in Table 1. An independent verification of these is to use them to derive the parameters of the glitch that soon followed the October 1997 event, and compare them with results obtained at radio frequencies. For this glitch, Wong et al. (2001) have publish more detailed parameters than Espinoza et al. (2011); therefore, their method of glitch analysis has been followed. We used their glitch epoch (MJD 50812.9) and their decay time scale (2.9 days) since the X-ray data is too sparse to derive these parameters independently. The X-ray data beyond the glitch epoch were analyzed using the model of Backer et al. (2000), and models 1 and 2 of Table 1 . The results are shown in Table 2; for comparison the corresponding values derived by Wong et al. (2001) are also shown (see their Table 3).

The errors on $t_{\mathrm{g}}$ and $\tau_{n}$ in Col. 2 of Table 2 cannot be used to estimate the errors in Cols. 3 to 5 , because the parameters of the fit are highly correlated, and the covariance matrix is obtained only when $t_{\mathrm{g}}$ and $\tau_{n}$ are also fit. The actual uncertainties are larger than those quoted in Cols. 3 to 5 of Table 2. However, the mean values of the derived X-ray parameters can be compared with the corresponding radio parameters and their errors.

From Table 2 it is clear that the glitch parameters derived using the three models are broadly consistent with each other. It can therefore be concluded that the post-event and pre-glitch solutions of Table 1 are fairly robust, and enhance the confidence in the results of the previous section.

\section{Discussion}

We conclude from this work that, strictly speaking, the X-ray data by itself cannot unequivocally discriminate between the two hypotheses, but offers reasonable evidence for intrinsic slowing down of the Crab Pulsar during the October 1997 event. However, when coupled with the very accurate radio reference timing model, the X-ray data strongly supports the intrinsic slowing down hypothesis. It may be worthwhile to check the Jodrell data for other occurrences of such slowing down in the Crab Pulsar.

Assuming that the Crab Pulsar indeed slowed down, the implications are the following:

1. The effect of such extreme events in pulsars with plerions must be taken into account while deriving their glitch parameters.

2. This is probably a slow version of the anti-glitch observed by Archibald et al. (2013) in a magnetar.

3. The explanation of Akbal et al. (2015) for the observed slowing down of PSR J1119-6127 after a glitch in May 2007 could be the mechanism driving the slowing down in the Crab Pulsar.

Acknowledgements. I thank Francis Graham-Smith for useful and encouraging discussion, and Andrew Lyne for verifying the X-ray timing residual behavior. I thank the referee for useful comments. This research made use of data obtained 
M. Vivekanand: The 1997 event in the Crab Pulsar in X-rays $(R N)$

from the High Energy Astrophysics Science Archive Research Center Online Service, provided by the NASA-Goddard Space Flight Center. It has also made use of data provided by the BeppoSAX satellite.

\section{References}

Akbal, O., Gugercinoglu, E., Sasmaz Mus, S., \& Alpar, M. A. 2015, MNRAS, 449, 933

Archibald, R. F., Kaspi, V. M., Ng, C. Y., et al. 2013, Nature, 497, 591

Backer, D. C., Wong, T., \& Valanju, J. 2000, ApJ, 543, 740

Cusumano, G. 2003, in Adv. Sp. Res. Proc. 34th COSPAR Scientific Assembly held in Houston [arXiv: astro-ph/0309615]

Espinoza, C. M., Lyne, A. G., Stappers, B. W., \& Kramer, M. 2011, MNRAS, 414,1679

Frontera, F., Costa, E., Dal Fiume, D., et al. 1997, A\&AS, 122, 357
Graham Smith, F., \& Lyne, A. G. 2000, in Pulsar Astronomy - 2000 and Beyond, eds. M. Kramer, N. Wex, \& N. Wielebinski (San Francisco: ASP), ASP Conf. Proc. 202, 499

Graham Smith, F., Lyne, A. G., \& Jordan, C. A. 2011, MNRAS, 410, 499

Hobbs, G. B., Edwards, R. T., \& Manchester, R. N. 2006, MNRAS, 369, 655

Jahoda, K., Swank, J. H., Giles, A. B., et al. 1996, Proc. SPIE, 2808, 59

Lyne, A. G., \& Graham Smith, F. 2006, Pulsar Astronomy, Cambridge Astrophysics

Lyne, A. G., Pritchard, R. S., \& Graham Smith, F. 1993, MNRAS, 265, 1003

Lyne, A. G., Pritchard, R. S., \& Graham Smith, F. 2001, MNRAS, 321, 67

Manchester, R. N., \& Taylor, J. H. 1977, Pulsars (W. H. Freeman)

Mineo, T. Cusumano, G., Kuiper, L., et al. 2000, A\&A, 335, 1053

Nicastro L., Cusumano, G., Lohmer, O., et al. 2004, A\&A, 413, 1065

Vivekanand, M. 2015, ApJ, 806, 190

Wong, T., Backer, D. C., \& Lyne, A. G. 2001, ApJ, 548, 447 